\title{
Shared population-level dynamics in monkey premotor cortex during solo action, joint action and action observation
}

\author{
Giovanni Pezzulo ${ }^{*}$, Francesco Donnarumma ${ }^{1 *}$, Simone Ferrari-Toniolo ${ }^{2,}{ }^{\ddagger}$, Paul Cisek ${ }^{3}$, \\ Alexandra Battaglia-Mayer²
}

1. Institute of Cognitive Sciences and Technologies, National Research Council, 00185 Rome, Italy

2. Department of Physiology and Pharmacology, SAPIENZA University of Rome, 00185 Rome, Italy

3. Department of Neuroscience, University of Montréal, Canada

* These authors contributed equally to this paper.

‡ Current address: Department of Physiology, Development and Neuroscience, University of Cambridge, Cambridge CB2 3EG, UK

Correspondence to:

Alexandra Battaglia-Mayer (alexandra.battagliamayer@uniroma1.it)

Giovanni Pezzulo (giovanni.pezzulo@istc.cnr.it) 


\begin{abstract}
Studies of neural population dynamics of cell activity from monkey motor areas during reaching show that it mostly represents the generation and timing of motor behavior. We compared neural dynamics in dorsal premotor cortex (PMd) during the performance of a visuomotor task executed under different contexts and during an observation task. In the former, monkeys moved a visual cursor in different directions by applying isometric forces on a joystick, either individually or in cooperation with a conspecific. In the latter, they observed the cursor's motion guided by the partner. We found that neural dynamics were shared across isometric and observation tasks and they discriminated directions more accurately than task types, suggesting that PMd encodes spatial aspects independently from specific behavioral demands. Therefore, the largest components of population dynamics might reflect higher cognitive processes, such as the representation of action goals or outcomes, rather than mechanisms strictly confined to motor functions.
\end{abstract}

Keywords: dorsal premotor cortex; action execution; action observation; joint action; population dynamics 


\section{INTRODUCTION}

Classical motor-related areas are involved not just in the specification of movement kinematics and/or dynamics ${ }^{1-3}$ but also in cognitive dimensions of behavioral tasks ${ }^{4-9}$. Motor cortex itself is considered a fundamental node in the processing of cognitive information related to motor acts, along with other cortical and subcortical structures that contribute to motor planning and execution ${ }^{10}$.

One of the most striking demostrations of the involvement of classical motor areas beyond purely motor tasks is the presence of covert representations of motor behavior without motor execution 11. Covert motor representations are evident in periods preceding memorized movements, when directional information is processed ${ }^{12}$ and during action observation. The mirror response found originally in neurons of ventral premotor cortex in monkeys 13-15 expresses at single cell level the match of the neural mechanisms involved in the observation of a given action with those engaged when the observer performs the same action.

These matching operations have been shown not only during overt motor performance (e.g. when observing an agent grasping a piece of food ${ }^{14,15}$ ) but also in more abstract contexts, involving the observation of a visual scene associated to a well-learned motor task, as when merely looking on a monitor a moving cursor, known to be controlled by an agent through a joystick ${ }^{16,17}$. It has been proposed that, while observing sensory stimuli strongly associated to subsequent motor actions, a mental rehearsal of the motor actions occurs at the neural level, which involves operations similar to those associated to overt performance ${ }^{16,17}$.

So far, the similarity of such matching operations, or the heterogeneity of context-dependent modulations of neural activity, have been mainly highlighted at a single cell level, using a traditional approach that focuses on the pattern of neural activation and its relation with movement parameters. In this study, instead, we adopt a dimensionality-reduction technique (Principal Component Analisis, PCA) to analyze the temporal evolution of patterns of neural activity in dorsal premotor cortex of the macaque brain, recorded during three different tasks. The first two tasks involve performing isometric actions to move a visual cursor to one of eight spatial targets arranged in a circle, in individual (SOLO) and dyadic (TOGETHER) conditions. The third task consists in the observation of the motion of a visual cursor generated by the action of another monkey (OBS-OTHER). The dimensionality-reduction approach used here permitted us to compare the temporal evolution of the system states - or trajectories in "neural space" 18 - for the 3 tasks and the 8 different spatial targets. Notably, while other studies measured neural population dynamics during one's own action planning and execution ${ }^{19}$, our experimental paradigm allowed comparing them with population dynamics during an action observation task that does not require action generation.

We addressed two main open questions. First, we asked how task-related variables (e.g., movement initiation, direction of cursor's motion, task identity) are coded at the population level in monkey premotor cortex. Second, we asked whether the same population-level coding is shared across tasks having different behavioral demands - and especially across tasks that require (SOLO and TOGETHER) or do not require (OBS-OTHER) overt action generation.

\section{RESULTS}

A principal component analyses (PCA) - or "neural space" analysis 20-22 - was conducted on the neural activity of a population of 384 premotor cells, obtained from a larger dataset of 471 neurons from dorsal premotor cortex (PMd; areas F7/F2; part of area 6; Fig. 1A), recorded from two monkeys (200 neurons in monkey K; 184 neurons in monkey S), during the execution of different visuomotor tasks. Within the same sessions, the monkeys performed 
two isometric hand force tasks in different contexts (SOLO and TOGETHER) and an observation task in absence of dynamic force production (see Figure 1 and Methods).

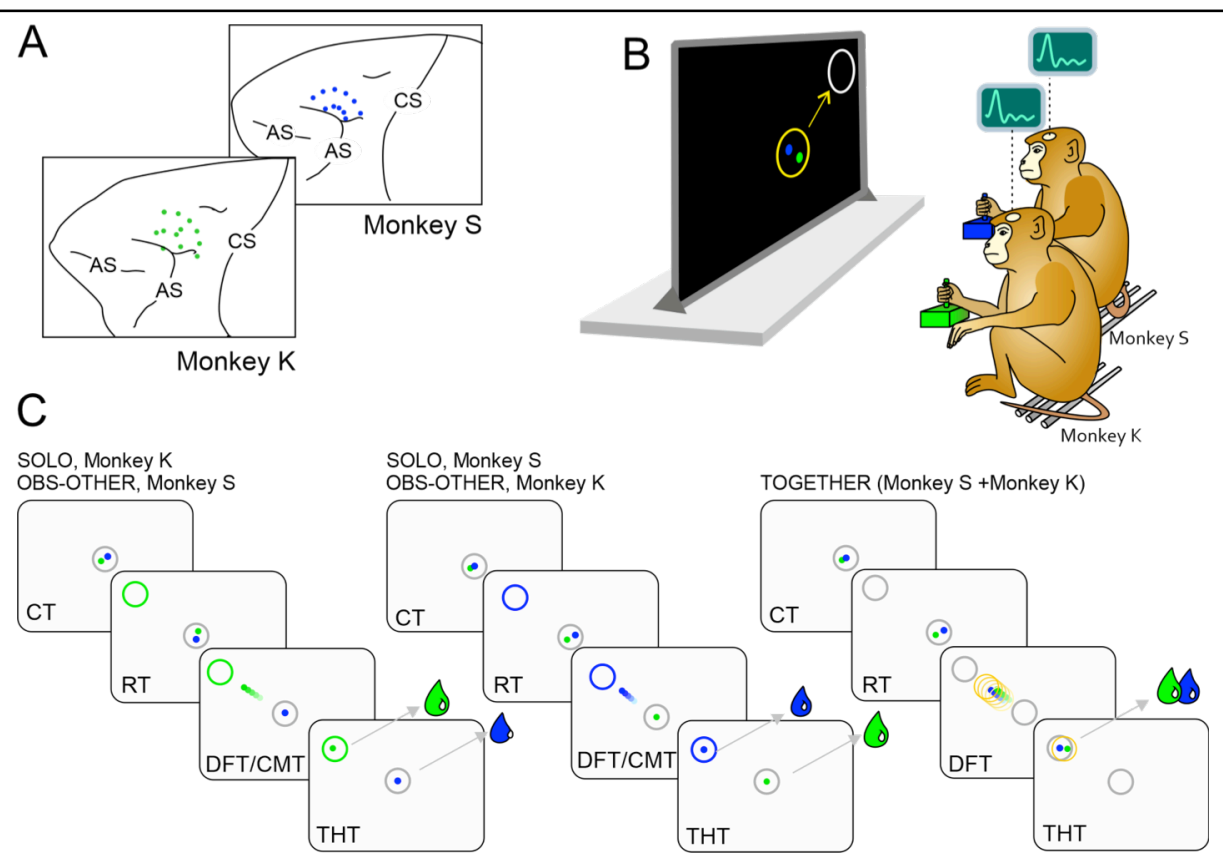

Figure 1. Recording sites, experimental apparatus and tasks. $\boldsymbol{A}$. Recording sites in areas F7/F2 of Monkeys S and K. B. Monkeys S and K sat next to each other and controlled through an isometric joystick their own cursors (S, blue dot; K, green dot) displayed on a screen. $\boldsymbol{C}$. At the beginning of each trial, the monkeys were required to bring their cursors from an offset position (not shown) to the central white circle (grey in C) for a variable Control Time (CT). In the SOLO task, each monkey, instructed by the color of one peripheral target ( $\mathrm{S}$, blue; $\mathrm{K}$, green) presented in one of 8 potential locations, had to bring its cursor on this target, in a subjective reaction time (RT), by applying individually a hand force pulse on the joystick to get a liquid reward. During the SOLO trial of one monkey, the other animal observed the moving cursor controlled by its mate (OBS-OTHER task) and was only required to actively maintain its cursor within the central circle until the end of the trial, to obtain its reward dose. In the SOLO and OBS-OTHER trials, the reward was delivered to each animal based on its individual (successful) performance, independently of the partner' behavior. In the TOGETHER trial, instructed by the white color of the peripheral target ( $\boldsymbol{B}$, grey in $\boldsymbol{C}$ ), both monkeys had to act jointly, by coordinating their force output in direction and timing, to guide together a common yellow circle from the center to the peripheral target to get both their reward doses. For the successful performance of the task, the animals had to keep their own cursors within a maximal inter-cursor distance for the entire Dynamic Force Time (DFT) application and until the end of the Target Holding Time (THT). Lack of inter-subject coordination resulted in unsuccessful trials and neither animal was rewarded. Notice that the DFT of the SOLO trials of one monkey corresponds to a cursor's motion time (CMT) interval of the OBS-OTHER task of the other animal.

The single-unit activity of the 471 cells recorded under the same task conditions was analysed in a previous study ${ }^{23}$, where a detailed description of the behavioral tasks is reported. In brief, two monkeys were sitting in front of a large monitor (Fig. 1B), and each held in its hand an isometric joystick to control its own visual cursor (blue for monkey S; green for monkey K). In the isometric tasks (Fig. 1C), the animals were instructed, by the color of a peripheral target, whether to bring their cursors from the central position to that target, individually (SOLO, blue or green target; Fig. 1C), or acting jointly with their companion (TOGETHER, white target; Fig. 1C). The latter condition required coordinating their forces to guide a common object (yellow circle) from the center to its final target location. During the TOGETHER condition, the instantaneous position of the moving yellow circle coincided with the midpoint of the coordinates of the two cursors, controlled simultaneously by the two animals. During 
the execution of the SOLO trials of one monkey, the partner was required to statically maintain its own cursor in the central position, without any dynamic hand force application, while observing on the screen the cursor's motion controlled by its partner (OBS-OTHER trials), (Fig. 1C). Therefore, the neural activity recorded from each animal was studied during the performance of three different tasks, SOLO, TOGETHER and OBS-OTHER.

\subsection{Single cell analysis}

The animal behavior in the three different tasks is described in detail in previous studies ${ }^{23,24}$. As already reported $23,66 \%$ (311/471) of our original dataset of cells were modulated at least in one of the three tasks, and 39\% (186/471) in more than one task. As expected, the spatial structure of the task highlighted the directional nature of the neural activity in all task conditions (Fig. 2A-B). In Fig. 2A we show an example of neural activity of a single cell which was significantly modulated, even though differently, during the SOLO and TOGETHER tasks (2w ANOVA, $\mathrm{p}=2.49 \mathrm{e}-17$, followed by the Bonferroni post hoc test). In Fig. 2B we show another neuron mainly active during the OBS-OTHER task. For each cell, we report in the form of rasters and spike density functions the activity recorded in the three task conditions (SOLO, green; OBS-OTHER, blue; TOGETHER, red), in the 8 directions of cursor's motion.
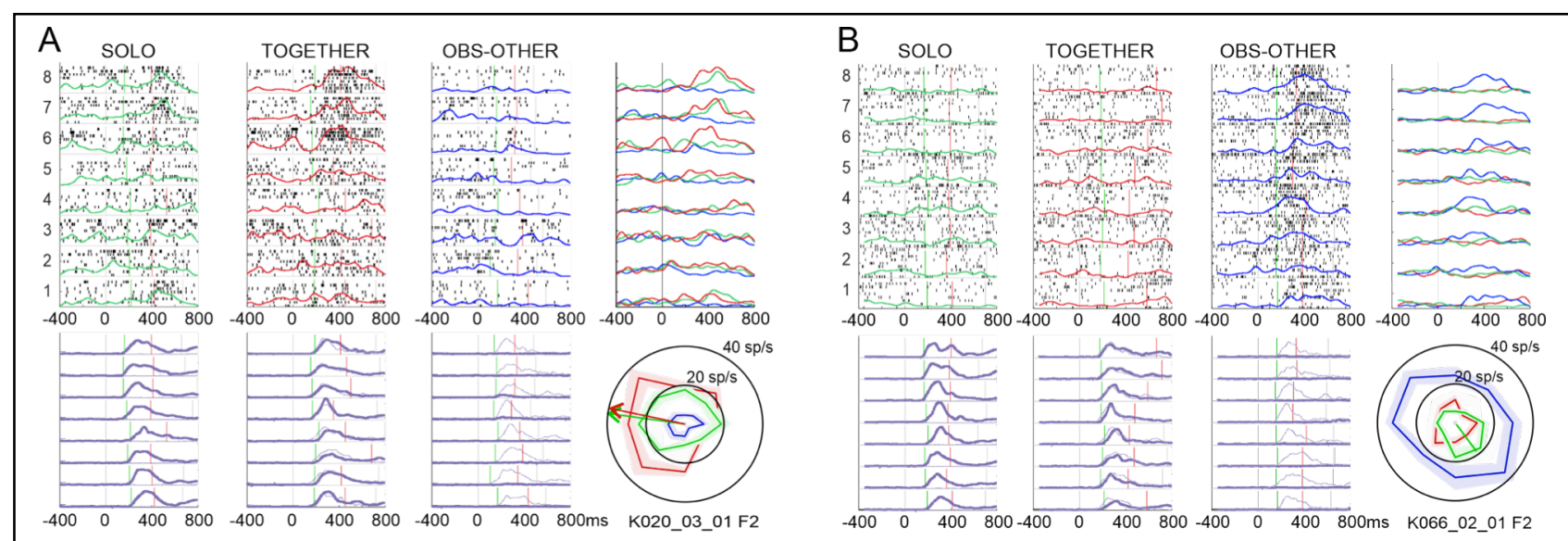

Figure 2. Examples of cell activity recorded in premotor cortex $\boldsymbol{A}$. Neural activity of a cell modulated during the isometric force application. The cell is active, even though differently (2w ANOVA, $\mathrm{p}=2.49 \mathrm{e}-17$, followed by the Bonferroni post hoc test), during both the SOLO and TOGETHER tasks, and not during the OBS-OTHER trials. $(\boldsymbol{B})$ Activity of another cell preferentially modulated during the OBS-OTHER task, relative to the two isometric tasks. In $\boldsymbol{A}, \boldsymbol{B}$ the neural activity is plotted for directions 1 to 8 in the form of raster plots, where each dot represents an action potential, as well as spike desity functions for each direction. The spike density functions calculated for the three tasks are overlapped (SOLO, green; TOGETHER, red; OBS-OTHER, blue) for direct comparison. The activity is also reported in the form of polar plots, where the mean firing rate computed during the DFT (for SOLO and TOGETHER) or the corresponding epoch (CMT) for the OBS-OTHER trials is plotted in the 8 directions, to highlight the directional nature of neural activity. For each cell, the animal's behavior is reported by showing, below the raster-plots, the corresponding speed profiles (purple curves) of the cursor guided by the monkey from which the spiking activity was recorded (thick curve) or by its partner (thin curve). In all panels, $0 \mathrm{~ms}$ corresponds to target onset, while green and vertical bars indicate cursor's motion onset and the time of its arrival on the final location, respectively.

\subsection{Neural space analysis: first four principal components}

We conducted two PCA analyses, on neural data aligned to target onset and to cursor's motion onset, respectively. For each analysis, we identified the four highest-ranked principal 
components X1-X4 (i.e., those explaining more variance), which captured $26.1 \%$ of the variance when aligned on target onset, and 34\% when aligned on cursor motion onset. We aggregated their activity according to the three tasks (i.e., SOLO, OBS-OTHER and TOGETHER; Fig. 3) or to the eight directions of cursor's motion (Dir 1-8; Fig. 4). Note that in the two motor tasks (SOLO and TOGETHER) "direction" may refer to both the direction of isometric force output and the direction of cursor's motion, but in the non-motor task (OBS-OTHER) only the latter applies. Here, we use "direction" to refer to the direction of cursor's motion, which applies to all the tasks. In both Figs. 3 and 4, panels A and B show the alignment to target onset and cursor's motion onset, respectively.

\section{Analysis of the four highest-ranked components aggregated by task type}

The profiles of the two highest-ranked principal components X1-X2 obtained by clustering across task conditions (SOLO, OBS-OTHER and TOGETHER; Fig. 3) are highly congruent across action types and express mainly the dynamical aspects of the tasks. The first component X1 shows a "state change" at target onset, whose peak is locked to the time of action initiation, occurring on average $220 \mathrm{~ms}$ after target presentation. The second component X2 shows a separate dynamical component, with a bell-shape profile evolving during the planning phase and peaking (independently on the task) at about $100 \mathrm{~ms}$ after target onset.
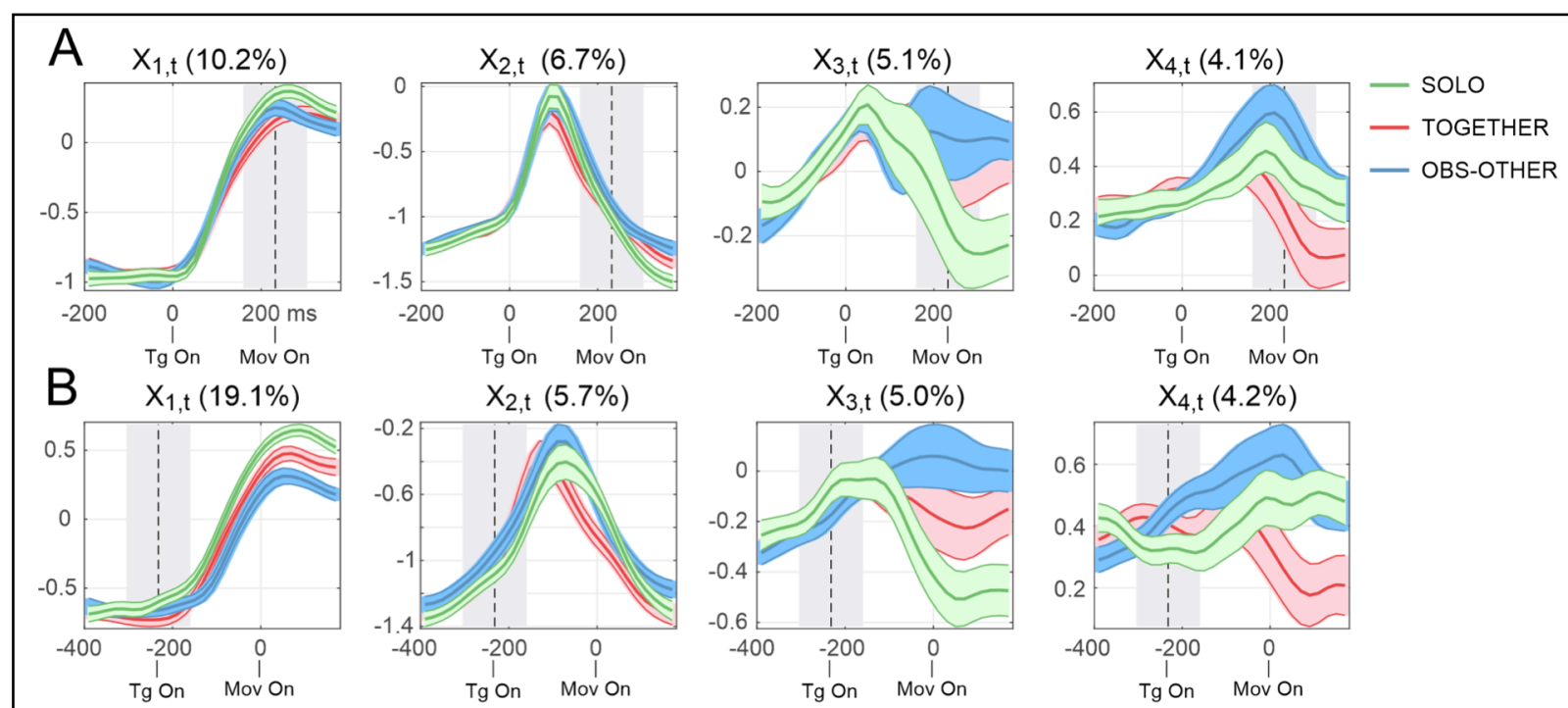

Figure 3. First four components of PCA aggregated according to the three task types. The four highestranked PCs, which captured in total 26,1\% (plot A) and 34\% (plot B) of the firing rate variance are plotted, after being aggregated relative to the 3 task to be performed (SOLO, OBS-OTHER and TOGETHER). The curves are obtained after aligning ( $0 \mathrm{~ms}$ ) the neural activity to target onset (TgOn) $(\boldsymbol{A})$ or cursor's motion onset (Mon On) $(\boldsymbol{B})$. The dotted vertical line and grey zone indicate the mean and variance (+/-SD) values of cursor's motion onset $(\boldsymbol{A})$ and target onset $(\boldsymbol{B})$, respectively. Given the bootstrap procedure adopted to plot the the graphs, the non-overlapping portions of neural space trajectories lies in the 5\%-95\% percentiles of the distribution of resampled differences, and consequently they can be considered significantly distinct and discriminable at $\mathrm{p}<0.05$.discriminable at $\mathrm{p}<0.05$.

While components $\mathrm{X} 1$ and $\mathrm{X} 2$ are similar between conditions, task type can be discriminated from them, particularly during cursor's motion. The distinction between tasks becomes clearer when the neural activity is aligned to MT onset (Fig. 3B). This finding indicates that 
while dynamical aspects are predominant, task-dependent aspects are jointly expressed with them in the two highest-ranked principal components.

The two components X3 and X4 discriminate task types even more clearly, especially around cursor's motion onset. Notably, while components X1, X2 and X4 peak well after the target presentation, component X3 shows a different profile: its amplitude starts increasing before target presentation and peaks few tens of milliseconds after it, suggesting that it may be related to the anticipation of target onset which served as the 'go' signal for task initiation.

\section{Analysis of the four highest-ranked components aggregated by directions of cursor's motion}

The profiles of the two highest-ranked components X1-X2 express primarily the dynamical aspects of the tasks even when clustering across directions of cursor's motion (D1-D8; Fig. 4). However, directional aspects can also be clearly discriminated in both. While in X1 the future direction of the cursor's motion can be discriminated around the time of movement onset, in $\mathrm{X} 2$ it can be discriminated about $100 \mathrm{~ms}$ before it, for both task alignments. Finally, the components X3 and X4 provide very effective information to discriminate all directions, even before cursor's motion onset (see below Section 2.4).

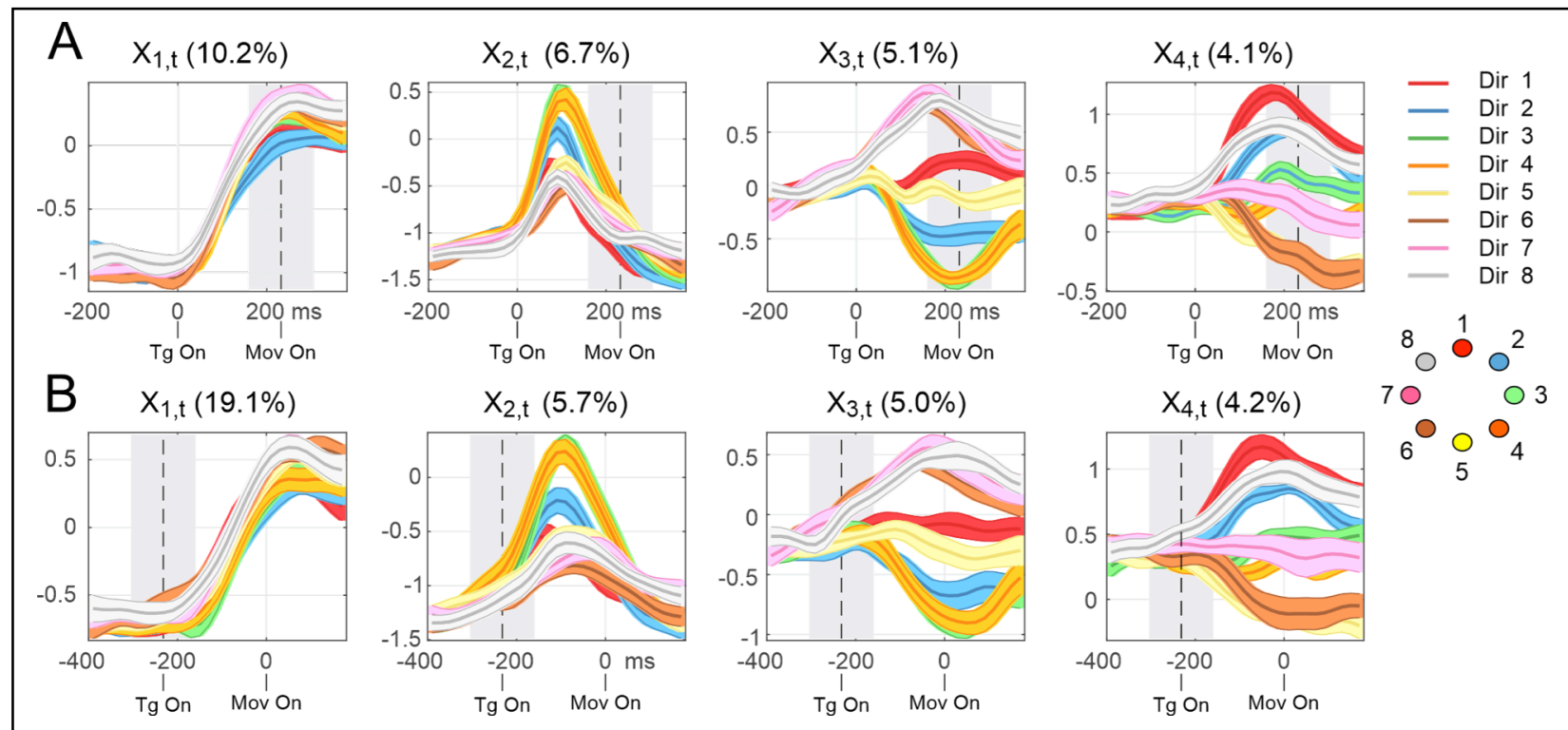

Figure 4. First four components of PCA aggregated according to the eight cursor's motion directions. The four highest-ranked PCs, which captured in total 26,1\% (plot A) and 34\% (plot B) of the firing rate variance are plotted, after being aggregated relative to the 8 directions of cursor's movement (Dir 1-8). The curves are obtained by aligning $(0 \mathrm{~ms})$ the neural activity to target onset (TgOn) $(\boldsymbol{A})$ or cursor's motion onset (Mov On) (B). Conventions, symbols and statistical procedures as in Fig. 3.

\subsection{Distance among trajectories in 12D neural space}

\section{Comparison of RMS (Root Mean Square) distances across directions and tasks}

To assess the relative importance of directional and task-related information, we analysed the neural trajectories in the 12D neural space, obtained from the first 12 components of PCA 
(X1-X12). Together, these components captured $41.3 \%$ and $39.6 \%$ of the firing rate variance when aligning neural activity to target onset and to cursor's motion onset, respectively.

We compared the Root Mean Square (RMS) distance in 12D neural space of 24 trajectories (corresponding to 24 behavioral conditions: 3 tasks $\times 8$ directions), with alignment on target onset. The Root Mean Square (RMS) distance is a standard measure of the average distance between pairs of trajectories; see the Methods for details. We performed multiple comparisons between pairs of trajectories that vary across task types and directions. For each trajectory associated to a specific task and direction (e.g. SOLO in direction D1; Fig. 5A) we calculate the distance, in any time bin, from i) the other 2 trajectories calculated in the same direction, but in the other two task conditions (i.e. SOLO in D1 vs. OBS-OTHER in D1 and TOGETHER in D1), and from ii) the other 7 trajectories calculated in the same type of task, but in different directions (i.e. SOLO in D1 vs SOLO in D2, SOLO in D3, ..., SOLO in D8; Fig. 5A). The results of the 9 comparisons for SOLO in direction D1, TOGETHER condition in D1, and OBSOTHER in D1 are plotted in Fig. 5A, 5B and 5C, respectively. The other comparisons (not shown) follow similar temporal profiles.
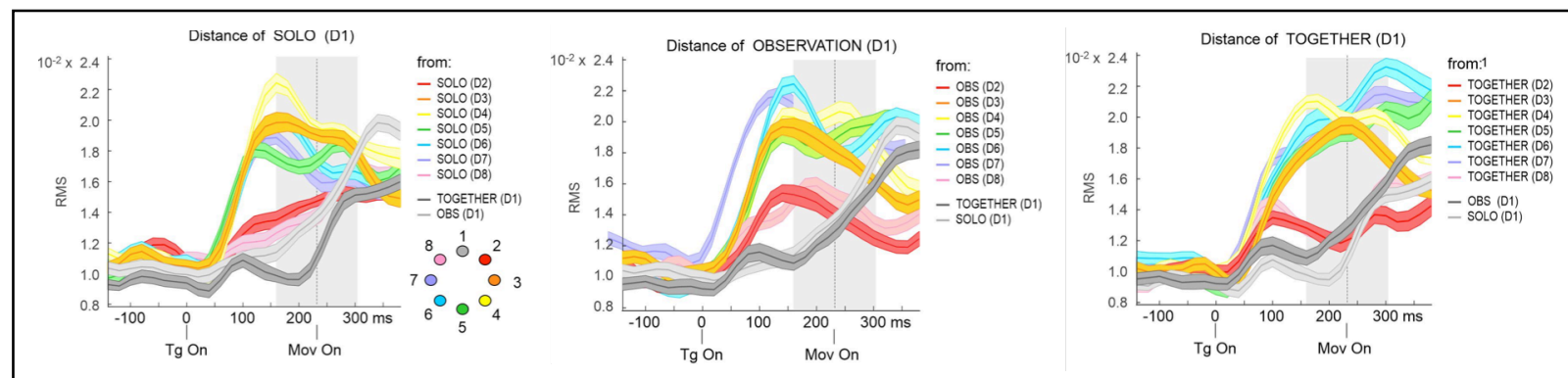

Figure 5. Example of comparisons of neural dynamics across tasks and directions. The comparisons are performed by computing the distance (RMS) in the 12D neural space, between pairs of neural trajetories which differ in the task (gray curves), or in the directions (colors). ( $\boldsymbol{A}$ ) RMS distances between the neural trajectory associated to SOLO condition in direction D1 vs. those differing for task but not direction (OBSOTHER in D1, light grey, and TOGETHER in D1, dark grey) and for direction but not task (SOLO in D2, SOLO in D3, etc.). (B-C) The same as in A, but showing the RMD distances from TOGETHER in D1 (B) and OBSOTHER in D1 $(\boldsymbol{C})$. In all instances, the alignment $(0 \mathrm{~ms})$ of activity is to target onset (TgOn). The dotted vertical line and grey shaded area indicate the mean and variance (+/-SD) values of cursor's motion onset, respectively.

RMS distances across both directions and tasks increase shortly (50-100ms) after target onset, but the former reach their peaks 100-150ms after target onset, well before cursor's motion onset, whereas the latter reach their peaks after cursor's motion onset. Importantly, neural trajectories in 12D neural space diverge more across directions of cursor's motion than across tasks, especially before movement onset, but (for most directions) also afterwards.

The highest RMS distances are obtained when comparing the same task across directions, especially those having an absolute angular difference greater than $45^{\circ}$ (D3-D7). Smaller differences emerge instead when comparing different types of actions, such as isometric force application vs. cursor's motion observation (SOLO vs OBS-OTHER), or when contrasting the same actions performed in different contexts (SOLO vs TOGETHER). 


\section{Comparison of RMS distances within motor tasks and between motor and non-motor tasks}

We next assessed whether RMS distance between OBS-OTHER and the two motor tasks (SOLO and TOGETHER) is greater than RMS distance within the two motor tasks. For this, we averaged all neural trajectories belonging to the same task and calculated their RMS distances over time. Figures 6 plots the RMS distance between SOLO vs. TOGETHER and OBS-OTHER (Figure 6A); between TOGETHER vs. SOLO and OBS-OTHER (Figure 6B); and between OBSOTHER vs. SOLO and TOGETHER (Figure 6C). The neural trajectories begin to diverge from one another within $100 \mathrm{~ms}$ after target onset, in the same way across comparisons, until the onset of cursor's motion time. At this point, the divergence amplitudes start to differ for the three comparisons: SOLO diverges similarly from the other two task, whereas TOGETHER and OBS-OTHER are more different from one another than they are from SOLO.

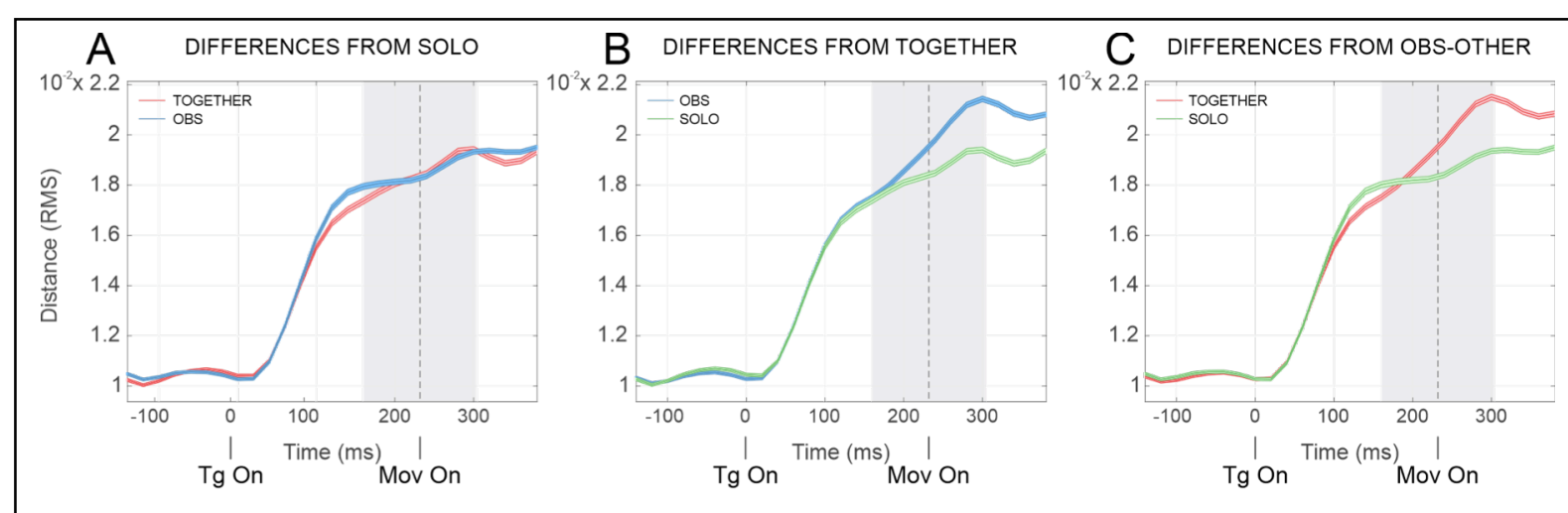

Figure 6. Distances (RMS) in 12D neural space between tasks. The comparisons are performed by firstly averaging all the neural trajectories belonging to the same task and then computing their distances (RMS) in the 12D neural space. (A) RMS distance between SOLO vs. TOGETHER and OBSOTHER tasks. (B) RMS distance between TOGETHER vs. SOLO and OBS-OTHER tasks. (C) RMS distance between OBS-OTHER vs. SOLO and TOGETHER tasks. Alignment ( $0 \mathrm{~ms}$ ) is to target onset (TgOn). The dotted vertical line and grey zone indicate the mean and variance (+/-SD) values of cursor's motion onset, respectively.

In sum, the analysis of RMS distances in 12D neural space indicates that neural trajectories diverge earlier and to a greater extent across directions cursor's motion than across task types. Furthermore, neural trajectories do not diverge more between motor and non-motor tasks than within motor tasks, despite the different behavioural demands between motor and non-motor tasks.

The analysis of RMS distances across directions suggests that these distances may not be arbitrary, but rather reflect a gradient of distance in physical space between targets. For example, in Figure 5A, the RMS distance between SOLO-D1 and its two "neighbours" in space (SOLO-D2 and SOLO-D8) remains relatively small, whereas it increases more steeply for D3D7. Our next analysis assesses the possible isomorphism between distances across directions in neural and physical spaces.

\subsection{Analysis of the topographical order of directional target encoding}

We asked whether a topographical order exists in the representation of the directions of cursor's motion in neural space, and whether it may reflect constraints of the external world, such as the spatial distance between the eight peripheral targets. 
For each of the three tasks, we plotted the (average) neural trajectories for the 8 directions, in the 2D space formed by the two PCA components that are more direction-sensitive: X3 and X4, which together explained $9.2 \%$ of the variance, when aligning the activity to both target onset (Fig. $7 \mathrm{~A}$ ) and on cursor's motion onset (Fig. 7B). In all panels, circles, crosses and squares indicate the trajectory points corresponding to the starting, alignment and ending time adopted for plotting the curves, respectively (see Fig. 7 legend).

In all the three tasks, the neural trajectories are clustered together in a common space before target presentation (circles; Fig. 7A), as previously reported in studies using a delay period ${ }^{18}$. All the trajectories diverge already around target onset (crosses; Fig. 7A), well before cursor's movement onset (crosses; Fig. 7B). Interestingly, the neural representation of cursor's motion in the 8 directions in neural space has a topographical structure. Neural trajectories are organized according to a directional gradient - which we refer to as "neural clock" - that is isomorphic to the spatial arrangement of target positions in the external world. In other words, targets that are closer in external spatial coordinates (e.g., D1 and D2 but not D6) are closer in neural space, too.

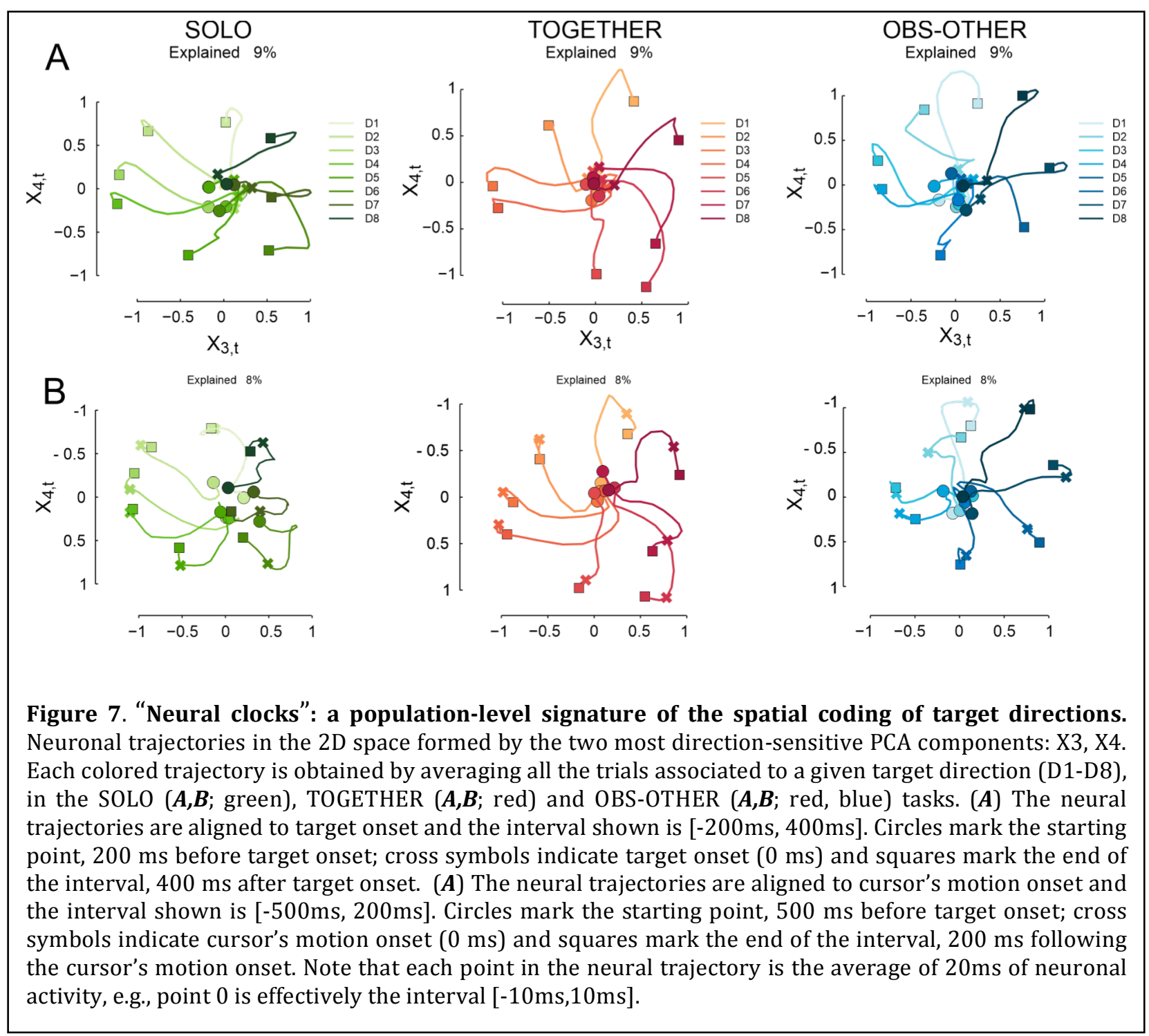


Intriguingly, the same (counter-clockwise) directional gradient is present not only in the two motor tasks, but also during action observation, when monkeys do not control the cursor's motion or apply any hand force. A control analysis of activity during a "saccade only" task, rules out the possibility that this similarity is due to oculomotor behavior, which is similar across the three tasks (see Supplementary Materials, Figs. S2 and S3).

\section{DISCUSSION}

We studied population-level dynamics in the premotor cortex of monkeys that performed two isometric tasks and an observation task. During the isometric tasks, monkeys applied a hand force on an isometric joystick to guide a visual cursor from a central position toward one of eight visual targets, individually (SOLO) or together with another monkey (TOGETHER). During the observation task, each animal observed the moving cursor controlled by its partner (OBS-OTHER), when the latter perfomed its SOLO trials.

We used a dimensionality reduction (PCA) analysis to ask (i) how task-related variables (e.g., action initiation, cursor's motion direction, task type) are encoded at the population level and (ii) whether this population coding differs when contrasting different behavioral demands, such as the two motor tasks (SOLO and TOGETHER) versus the non-motor task (OBS-OTHER).

\subsection{First components signal shared dynamics across behavioural tasks}

The multi-task approach of this study afforded different comparisons of the neural dynamics across several cognitive-motor conditions. In particular, we contrasted: i) similar motor tasks performed in different contexts (e.g. SOLO vs TOGETHER); ii) action execution and observation (e.g. SOLO vs OBS-OTHER); iii) same type of action performed in different directions (e.g. SOLO in eight directions); and iv) observing the consequences of the other partner's action, i.e. a visual cursor's motion, toward different locations (i.e. OBS-OTHER).

The analysis of the highest-ranked principal components that emerged from PCA (i.e., those explaining the most variance) showed that in premotor cortex a large portion of the neural variance was shared (i.e., had similar dynamics) across the three tasks. This is despite the fact that the three tasks involved striking differences in behavioral demands: the two motor tasks (SOLO and TOGETHER) have different control demands (individual vs. collaborative control) and provide different visual inputs during their performance (e.g. one cursor vs. two cursors with a circle); and the differences between motor (SOLO and TOGETHER) and non-motor (OBS-OTHER) tasks are even more significant.

Previous studies of neural population dynamics in motor and premotor cortex that used dimensionality reduction (like PCA) also found the highest ranked components to be related to dynamical aspects of the task; and interpreted them as related to state changes and movement timing, rather than movement type ${ }^{25}$. Views of motor cortical activity operations regarded as a dynamical system have proposed that a major proportion of premotor activity is devoted to signaling phase dynamics and in particular preparatory states, reflecting the initialization of a dynamical system at optimal conditions, which will later generate a movement 18,26,27. Our findings confirm the predominance of dynamical aspects in the two highest-ranked components of PCA (X1 and X2), but offer a new neurophysiological interpretation compared to those proposed so far in the literature. 
Dynamical aspects of neural space do not necessarily reflect an internal translation from movement preparation to its generation

The similarity of population-level coding across motor and non-motor tasks may help in contextualizing the interpretation of previous findings about action coding in premotor cortex, and especially the roles of the dynamical aspects of neural space found to be encoded in the highest-ranked PCA components. Our findings are in fact consistent with previous works using a range of techniques, including the orthonormalized neural trajectories of a Gaussian Process Factor Analysis shown in $\mathrm{Yu}$ et al. ${ }^{22}$, the Canonical Correlation Analysis (CCA) projections of the neural population response and the modeled data 28 and the dPCA components of Kaufman et al. ${ }^{25}$. As a reminder, the CCA attempts to find the patterns which are common across two data sets, such that the reweighted data sets (i.e. canonical variables) are maximally correlated.

In $\mathrm{Yu}$ et al. ${ }^{22}$ and following studies 25,29 , the interpretation of dynamical aspects of neural space is that their pattern is "presumably related to generating the arm movement and it is thus sensible that it is time-locked to movement onset". However, in the above studies, the results were obtained only from neural data collected during motor performance in a classical delayed-reaching paradigm. As these studies did not investigate the dynamics of forms of behavior not requiring movement generation, the proposed interpretation might have been biased by the nature of the adopted experimental paradigm.

Here, for the first time, we considered also a condition requiring monkeys to merely observe the consequences of the motor output of another monkey. Our findings indicate that in premotor cortex the same dynamics found in two isometric tasks (SOLO and TOGETHER) is shared with a behavioral condition which did not involve a motor act, but rather its abstract representation (OBS-OTHER). The fact that action observation leads to a pattern of highestranked components $\mathrm{X} 1$ and $\mathrm{X} 2$ similar to those associated to action requiring hand force application offers a different perspective on their roles - which goes beyond their reiterated attribution to movement generation $22,25,29$, which was also predicted by theoretical models ${ }^{28}$. Kaufman et al. ${ }^{25}$ suggested that highest-ranked components reflect an internal transition from movement preparation to movement generation. Our data in the OBS-OTHER condition suggest that the transition does not necessarily imply a physically performed movement, but also an internally simulated action.

Alternatively, one may consider the temporal profile of highest-ranked components found in our and other studies to correspond to "condition-independent" dynamics - a view consistently proposed to interpret the results of previous studies in different cortical areas and different tasks ${ }^{25,29-31}$. Condition-independent components have been regarded ${ }^{30}$ as the building blocks of neural activity, which capture its temporal modulations throughout the trial; and similar to our study, they are mostly locked to stimulus presentation. In this study, it is plausible that these components replicate the analogous sensory-motor temporal structure shared in the three behavioral conditions (SOLO, TOGETHER, OBS-OTHER). The dynamics captured by the pattern of the two highest-ranked components might therefore mirror the occurrence or the expectancy of behavioral events (e.g. central target appearance, followed by a peripheral target presentation, which in all instances predicts a cursor's motion), or more simply the presentation of a task-relevant stimulus - which are shared across tasks. Finally, we cannot exclude that the condition-invariance of the highest-ranked components might relate to decision dynamics occurring between the peripheral target presentation and cursor's motion onset, which select the type of action and the direction of the (real or internally simulated) force application. In this perspective, the neural dynamics extracted from the higher-order components may reflect evidence accumulation ${ }^{32}$ or an urgency signal 
33,34 that guides the choice between different action types, rather than motor generation. These and other alternative hypotheses remain to be fully explored in future studies.

It is worth noting that we can exclude that the above described dynamics can be attributed to eye behavior, which was very similar in the three tasks 23,35 . The analysis of the highestranked principal components X1-X4 obtained by clustering across SOLO, OBS-OTHER, TOGETHER, and EYE tasks reveals that the neural representation of the EYE task is markedly different from the other three (supplementary Figures S2 and S3). To our knowledge, this is the first investigation of the putative influence of oculomotor behavior on the neural dynamics associated with motor behavior.

\subsection{Directionality encoding}

We found that that direction of cursor's motion and task identity can be decoded prior to movement onset in the highest-ranked components and intertwined with phase dynamics (Fig. 3-4), rather than being only expressed in lower-ranked components. Our PCA-based analysis hence recovers the results of numerous previous studies that showed that spatial and motor parameters such as movement direction 36,37 and force can be accurately decoded from cortical populations ${ }^{38-40}$.

Interestingly, neural trajectories diverged more profoundly across directions of cursor's motion than across types of action for any given direction (see Fig. 5). This result suggests that spatial coding may consist of a neural process that operates separately and independently from a neural code aimed at distinguishing different types of action.

A rationale for this organization of neural space may lie in the functional specialization of premotor cortex, in which dynamical and spatial aspects of movement may need to be expressed independently from the specific task to be performed. In this perspective, a large population of neurons in premotor cortex may convey dynamical and kinematic features to shape motor representations that can subserve different contexts and tasks, including action planning, execution and observation. Smaller subpopulations of neurons (whose variance is captured by low ranked PCA components) may instead convey higher-order signals for taskand context-related information, which allow distinguishing amongst behaviors with similar temporal structure, like SOLO, TOGETHER and OBS-OTHER. This scenario is in line with a single neuron network topology, based on the existence of functional modules across cortical areas that guarantee fast and dynamical information processing and transfer ${ }^{41}$, and where the operations of even a small fraction of the network are sufficient to characterize a reasonable amount of the spatio-temporal features of neural activity.

Neural clocks: isomorphism between target direction representation in neural and external spaces, across motor and non-motor tasks

We found a remarkable isomorphism between the neuronal representation of movement directions and the arrangement of targets in the external space. Similar to previous studies, the trajectories in the 2D neural space of Figure 7 start from a central, equi-potential (or noninformative) state and diverge at the target onset 18,42. However, remarkably, they follow directions in neural space that are isomorphic to the spatial position of external targets. This topographical organization ("neural clock") may be a potential population-level signature of the spatial coding of target directions. Interestingly, the "neural clock" emerges in all the three tasks; and has the same orientation with respect to the real physical space of targets. In contrast, it does not emerge in a control task consisting of saccadic eye movements to eight 
spatial targets, suggesting that it does not result from oculomotor dynamics (see Supplementary Materials).

\subsection{Conclusions}

In sum, the present results indicate a novel sharing of population-level dynamics in monkey premotor cortex across motor and non-motor tasks, despite their different behavioral demands. The sharing becomes evident by considering the similarity of the highest-ranked components and of the "neural clocks" across the three tasks; and the fact that trajectories in 12D neural space do not diverge more between motor and non-motor tasks than across motor tasks. These findings suggest that the largest components of population dynamics in premotor cortex express dynamical and spatial aspects of movement independently from the task to be performed. They may reflect an abstract, covert representation of action rather than mechanisms strictly confined to motor functions.

The sharing of action representations across motor and non-motor task reported in this study is consistent with a large body of literature. First, single cell data analysis of our database showed similarities of the neural activity recorded during visual observation and isometric hand action trials ${ }^{23}$, with one third of the original dataset consisting of cells modulated during both observation and at least one of the two isometric action tasks. Despite the heterogenity of neural responses, quantified on the basis of their firing rates, some cells were similarly modulated or shared directional tuning across task conditions. Furthermore, our findings are reminiscent of mirror-like mechanisms, possibly supporting a matching operation between action execution and observation 14,15,43. We note, however, that because we used isometric tasks, what monkeys actually observed are the consequences of the companion's actions, not their overt movements. The putative matching between performed and observed actions occurs at the level of the cursor's motion, not of limb movements - similar to the shared representation found in premotor neurons in abstract contexts akin to ours ${ }^{16,17}$. Finally, our results are in keeping with the finding that dorsal premotor cortex preferentially codes target position and not limb movement during a delay period ${ }^{44}$; see also ${ }^{45}$. Our study suggests that a similar mechanism could operate during reaction-time tasks, without explicit delay periods. Previous evidence of similarities across motor and non-motor conditions were interpreted in terms of movement suppression 46 or action simulation ${ }^{11}$. The present study was not designed to disentangle between these (or other) possibilities, which require further investigation. 


\section{ONLINE METHODS}

\subsection{Animals and tasks}

Two adult male rhesus monkeys (Macaca mulatta; monkey $S$ and monkey K; body weight 7.5 and $8.5 \mathrm{Kg}$, respectively) were trained in pairs to perform an isometric hand force center-out task, in two different conditions: 1 ) individually (SOLO) or ii) in a joint-action context, where each animal had to coordinate its force with its companion to achieve a common goal (TOGETHER). During the recording sessions, the two animals sat next to each other (Fig. 1B), in front of a 40 -inch monitor at a distance of $150 \mathrm{~cm}$ from the eyes. Experimental and surgical procedures were performed in conformity with European Directive (63-2010 EU) and Italian (D.L. 26-2014) laws on the use of nonhuman primates in scientific research, and under authorization of the Ministry of Health of Italy to Alexandra Battaglia-Mayer. During the experimental procedures, all efforts were made to minimize animal suffering. Details about surgical procedures have been reported previously ${ }^{23}$.

Details about the behavioral tasks have been already reported in ${ }^{23,24}$. Briefly, each monkey applied a hand force on an isometric joystick (ATI Industrial Automation, Apex NC), to control and move a visual cursor in a center-out task, from a central position toward on one of the 8 peripheral targets, placed at an eccentricity of 8 degrees if visual angle (DVA). The joystick consisted of a force-transducer which measured the forces in two dimensions (Fx, Fy), in absence of any hand/arm displacement. To bring the cursor from the center to the peripheral targets a force pulse of about $3.2 \mathrm{~N}$ was required. Each monkey was trained to control its own colored cursor (blue for Monkey $S$ and green for Monkey K; diameter 0.6 DVA. The neural activity was recorded when the animals performed the isometric task under two different conditions (SOLO and TOGETHER trials), as well as when they observed (OBS-OTHER trials) the motion of the visual cursor controlled by the other animal (namely, observing the SOLO trials of the other monkey), without any dynamic force application. During the experiment, the animals used the right arm, contralateral to the recording chamber, while their left arm was gently restrained.

In the isometric SOLO and TOGETHER tasks (Fig. 1C), trials started with the appearance of a central white circle. The animals had to bring their own cursors within it from an offset position, keeping them there for a variable control time (CT, 500-600 ms), by exerting a small static hand force. Then, a peripheral target (outlined circle, 2 DVA in diameter) appeared in one of eight possible locations. The color of the target circle indicated which of the two animal had to move the cursor (blue: SOLO for monkey S; green: SOLO for monkey K, white: TOGETHER, both monkeys), hence the type of task (SOLO, TOGETHER and OBS-OTHER) to be performed (Fig. 1B). In the SOLO trials, one monkey performed the task individually, by guiding its own cursor from the center toward the peripheral target (Fig. 1C), to obtain a liquid reward. When one animal moved its cursor in a SOLO condition, its companion had to keep its own cursor inside the central target, in order to get a liquid reward, until the end of the SOLO trial of the other monkey. During this time, the companion animal observed the partner's action resulting in the motion of a cursor on the screen,therefore the companion animal's trials are referred as 'OBS-OTHER' trials (Fig. 1C). Note that this setup is different from other action observation studies. In fact, the isometric nature of the task did not provide the acting monkey and its its partner any other visual cue on the performed action, than the resulting motion of a visual cursor on the screen. In both SOLO and OBS-OTHER trials, the reward delivery to each animal depended only on the success of its own performance, which was independent from the partner's behavior. Although controlled (see below), no constraints 
were imposed on eye movements, since under this condition we were interested in studying the natural oculomotor behavior of each animal.

Besides the SOLO condition, the isometric force application was tested also in the interactive context of the TOGETHER task. When the peripheral target was white in color, both monkeys were required to coordinate their force output in space and time to bring together a common visual object (yellow circle) (Fig. 1C), toward it. The yellow circle appeared simultaneously to the peripheral target in the center of the workspace. To succeed in their common goal and to prevent aborting the trial, the monkeys had to maintain a maximum inter-cursor distance limit, coinciding with the diameter of the yellow circle (IDmax, 5 degrees VA). The moving yellow circle controlled by the two animals was centered at any instant at the midpoint of the two cursors, and once it reached successfully its final location, both monkeys received simultaneously an equal amount of liquid reward. The amount of reward dispensed was identical across task conditions.

Trials were presented in an intermingled fashion and pseudo-randomized in a block of a minimum of 192 trials, consisting of 8 replications for each of the 8 target directions and the three distinct conditions (two SOLO conditions, one for each animal, which corresponded to OBS-OTHER condition for the non-acting monkey) and one TOGETHER condition (3 conditions $\mathrm{x} 8$ directions $\mathrm{x} 8$ replication=192).

Finally, a saccadic eye movement task (EYE) was used as a control condition to evaluate the influence of eye-related signals on the neural activity recorded during the other task conditions. In separate blocks of trials, each animal was required to fixate initially a central white square for a variable CT (700-1000 ms), and then to make a saccade toward one of 8 peripheral targets (8 DVA eccentrity), which appeared at the completion of CT. Eye position and movement were recorded through a non invasive infrared oculometer (Arrington Research).

\subsection{Electrophysiological recordings}

Single-unit activity was recorded from dorsal premotor cortex (PMd; area F7/F2; area 6; Fig. 1A) of the two co-acting monkeys. Neurophysiological recording performed during the abovementioned tasks resulted for each monkey in three sets of data, relative to the three tasks in which the animal behavior was tested (SOLO, TOGETHER, OBS-OTHER). The neural activity was recorded extracellularly using two separate 5-channel multiple-electrode arrays (Thomas Recording, Giessen, Germany) from the two brains. The activity of each unit was collected in blocks of 192 trials (corresponding to 8 directions x 3 task conditions x 8 replications). In each session (1-3 session/day), constituted by the block of 192 trials, we recorded on average 6 units/session in monkey S, and 7 units/session in monkey K, for a total of 36 experimental sessions.

\subsection{Data analysis}

\section{Cell modulation and directional tuning}

To assess that a cell was "directionally-tuned" we used the same method adopted in ${ }^{23}$, consisting of a bootstrap procedure ${ }^{47}$ aimed at evaluating the statistical significance of its directional tuning. The bootstrap was applied to the tuning strength (TS), defined as the amplitude of the mean vector expressing the firing rate in polar coordinates ${ }^{48}$. A shuffling procedure randomly reassigned single-trial data to different target directions for 1000 times and the TS from the shuffled data was determined. A cell was labeled as "directionally-tuned" 
in a specific epoch of one task, if the TS value calculated from the original unshuffled data was higher than the computed confidence limit $(\mathrm{p}<0.05)$.

\section{Neural space analysis}

We conducted a principal component analysis (PCA) - also called "neural space" analysis ${ }^{20-22}$ - on the the neural activity of a population of 384 premotor cells (200 neurons recorded in monkey K, 184 neurons in monkey S), obtained from a larger dataset of 471 neurons, whose activity have been analysed in ${ }^{23}$. The cells selected for the present study were those for which the activity was collected in at least 8 replications in all the 3 task conditions, in all the 8 cursor's motion directions.

The analysis was performed in two different time intervals, corresponding to different activity alignments: i) the interval spanning from $200 \mathrm{~ms}$ before (TgOn; $0 \mathrm{~ms}$ ) to $400 \mathrm{~ms}$ after target onset; ii) the interval from $500 \mathrm{~ms}$ before (CMon) to $200 \mathrm{~ms}$ after cursor's motion onset. In both instances we adopted a $20 \mathrm{~ms}$ time binning. It is worth noticing that in our task the peripheral target onset was informative both about the direction of the cursor's motion and the type of action to be performed (SOLO, TOGETHER, OBS-OTHER).

To assess the robustness of the population activity during different tasks and cursor's motion directions, we performed a bootstrap test for each neural space trajectory ${ }^{49}$. The bootstrap procedure consisted of resampling the firing rate data of each cell 10,000 times in each time bin and grouping them by tasks and directions, in order to produce a distribution of the difference in the mean trajectory at each data point. The resulting confidence intervals showed if and when in time the compared distributions were significantly different. Therefore, all the results shown in Figs. 3-6 obtained with a bootstrap procedure are such that the nonoverlapping portions of neural space trajectories lies in the $5 \%-95 \%$ percentiles of the distribution of resampled differences. Consequently, they can be considered significantly distinct and discriminable at $\mathrm{p}<0.05$.

To compare quantitatively the differences between the encoded variables (such as direction and task) we considered a 12D neural space, formed by the first 12 components X1-X12 emerging from the PCA. We adopted a distance metric between neural trajectories in this 12D space, using the Root Mean Square (RMS) of the Euclidean distance computed at each $20 \mathrm{~ms}$ time bin. In particular, given two neural trajectories $\mathbf{p}(t)$ and $\mathbf{q}(t)$, at each $20 \mathrm{~ms}$ time bin $t$, we computed the $\mathrm{K}=12$ coefficients of each trajectory projected into the $12 \mathrm{D}$ space, i.e., $\mathrm{c}^{\mathbf{p}}(\mathrm{t})=$ $\mathrm{cp}_{1}(\mathrm{t}), \ldots \mathrm{cp}_{\mathrm{k}}(\mathrm{t})$ and $\mathrm{cq}(\mathrm{t})=\mathrm{cq}_{1}(\mathrm{t}), \ldots \mathrm{cq}_{\mathrm{k}}(\mathrm{t})$, respectively. Thus, at each time step, we did define the quantity:

$$
R M S_{\mathbf{p q}}(t)=\sqrt{\frac{\sum_{k=1}^{K}\left(c p_{k}(t)-c q_{k}(t)\right)^{2}}{K}}
$$

Note that the RMS distance allows comparing different projections on different space dimensions, as it is normalized for the number of components. When we consider a number $\mathrm{N}$ (in our case $\mathrm{N}=10,000$ ) of bootstrap samples, we can straightforwardly compute for each time step a distance between sets of samples, $\mathbf{p} \in \mathrm{P}, \mathbf{q} \in \mathrm{Q}$ (e.g., samples belonging to different tasks and directions), by means of the following equation:

$$
R M S_{P Q}(t)=\sqrt{\frac{\sum_{i, j=1}^{N}\left(R M S_{\mathbf{p}^{i} \mathbf{q}^{j}}(t)\right)^{2}}{N^{2}}}
$$


bioRxiv preprint doi: https://doi.org/10.1101/2020.12.23.424150; this version posted December 23, 2020. The copyright holder for this preprint (which was not certified by peer review) is the author/funder, who has granted bioRxiv a license to display the preprint in perpetuity. It is made available under aCC-BY-NC-ND 4.0 International license.

Our analysis using RMS distance was based on 24 trajectories (3 tasks x 8 directions) in 12D neural space, with alignment on target onset (Figure 5).

\section{Acknowledgements}

This research received funding from the MIUR of Italy, Prin 2017 (Grant N. 201794KEER_002) to ABM; the SAPIENZA-University of Rome ("Ricerche universitarie 2019") to ABM; the European Union's Horizon 2020 Framework Programme for Research and Innovation under the Specific Grant Agreement Nos. 785907 and 945539 (Human Brain Project SGA2 and SGA3) to GP; and the European Research Council under the Grant Agreement No. 820213 (ThinkAhead) to GP. The authors are sincerely grateful to Prof. Roberto Caminiti for his valuable suggestions and remarks. 


\section{References}

1. Archambault, P. S., Ferrari-Toniolo, S. \& Battaglia-Mayer, A. Online control of hand trajectory and evolution of motor intention in the parietofrontal system. J. Neurosci. Off. J. Soc. Neurosci. 31, 742-752 (2011).

2. $\quad$ Caminiti, R., Johnson, P. B. \& Urbano, A. Making arm movements within different parts of space: dynamic aspects in the primate motor cortex. J. Neurosci. 10, 2039-2058 (1990).

3. Moran, D. W. \& Schwartz, A. B. Motor cortical representation of speed and direction during reaching. J. Neurophysiol. 82, 2676-2692 (1999).

4. Battaglia-Mayer, A., Caminiti, R., Lacquaniti, F. \& Zago, M. Multiple levels of representation of reaching in the parieto-frontal network. Cereb. Cortex N. Y. N 1991 13, 1009-1022 (2003).

5. Carpenter, A. F., Georgopoulos, A. P. \& Pellizzer, G. Motor cortical encoding of serial order in a context-recall task. Science 283, 1752-1757 (1999).

6. Crutcher, M. D., Russo, G. S., Ye, S. \& Backus, D. A. Target-, limb-, and context-dependent neural activity in the cingulate and supplementary motor areas of the monkey. Exp. Brain Res. 158, 278-288 (2004).

7. Georgopoulos, A. P. Neural aspects of cognitive motor control. Curr. Opin. Neurobiol. 10, 238-241 (2000).

8. Hepp-Reymond, M., Kirkpatrick-Tanner, M., Gabernet, L., Qi, H. X. \& Weber, B. Contextdependent force coding in motor and premotor cortical areas. Exp. Brain Res. 128, 123-133 (1999).

9. Omlor, W. et al. Context-dependent limb movement encoding in neuronal populations of motor cortex. Nat. Commun. 10, 4812 (2019).

10. Turner, R. S. \& Anderson, M. E. Context-Dependent Modulation of Movement-Related Discharge in the Primate Globus Pallidus. J. Neurosci. 25, 2965-2976 (2005).

11. Jeannerod, M. Motor Cognition. (Oxford University Press, 2006).

12. Ashe, J. et al. Motor cortical activity preceding a memorized movement trajectory with an orthogonal bend. Exp. Brain Res. 95, 118-130 (1993).

13. Di Pellegrino, Fadiga, L., Fogassi, L., Gallese, V. \& Rizzolatti, G. Understanding motor events: a neurophysiological study. Exp Brain Res 91, 176-180 (1992).

14. Gallese, V., Fadiga, L., Fogassi, L. \& Rizzolatti, G. Action recognition in the premotor cortex. Brain 119, 593-609 (1996).

15. Rizzolatti, G. \& Craighero, L. The mirror-neuron system. Annu. Rev. Neurosci. 27, 169192 (2004).

16. Cisek, P. \& Kalaska, J. F. Neural correlates of mental rehearsal in dorsal premotor cortex. Nature 431, 993-996 (2004).

17. Tkach, D., Reimer, J. \& Hatsopoulos, N. G. Congruent activity during action and action observation in motor cortex. J Neurosci 27, 13241-13250 (2007).

18. Shenoy, K. V., Sahani, M. \& Churchland, M. M. Cortical control of arm movements: a dynamical systems perspective. Annu. Rev. Neurosci. 36, 337-359 (2013).

19. Vyas, S., Golub, M. D., Sussillo, D. \& Shenoy, K. V. Computation Through Neural Population Dynamics. Annu. Rev. Neurosci. 43, 249-275 (2020).

20. Machens, C. K., Romo, R. \& Brody, C. D. Functional, But Not Anatomical, Separation of "What" and "When" in Prefrontal Cortex. J. Neurosci. 30, 350-360 (2010).

21. Nicolelis, M. A., Baccala, L. A., Lin, R. C. \& Chapin, J. K. Sensorimotor encoding by synchronous neural ensemble activity at multiple levels of the somatosensory system. Science 268, 1353-1358 (1995).

22. Yu, B. M. et al. Gaussian-Process Factor Analysis for Low-Dimensional Single-Trial Analysis of Neural Population Activity. J. Neurophysiol. 102, 614-635 (2009). 
23. Ferrari-Toniolo, S., Visco-Comandini, F. \& Battaglia-Mayer, A. Two brains in action: Joint-action coding in the primate frontal cortex. J. Neurosci. 1512-18 (2019) doi:10.1523/JNEUROSCI.1512-18.2019.

24. Visco-Comandini, F. et al. Do non-human primates cooperate? Evidences of motor coordination during a joint action task in macaque monkeys. Cortex 70, 115-127 (2015).

25. Kaufman, M. T. et al. The Largest Response Component in the Motor Cortex Reflects Movement Timing but Not Movement Type. eNeuro 3, (2016).

26. Fetz, E. E. Are movement parameters recognizably coded in the activity of single neurons? Behav. Brain Sci. 15, 679-690 (1992).

27. Churchland, M. M. et al. Neural population dynamics during reaching. Nature 487, 5156 (2012).

28. Sussillo, D., Churchland, M. M., Kaufman, M. T. \& Shenoy, K. V. A neural network that finds a naturalistic solution for the production of muscle activity. Nat. Neurosci. 18, 10251033 (2015).

29. Lara, A. H., Elsayed, G. F., Zimnik, A. J., Cunningham, J. P. \& Churchland, M. M. Conservation of preparatory neural events in monkey motor cortex regardless of how movement is initiated. eLife 7, e31826 (2018).

30. Kobak, D. et al. Demixed principal component analysis of neural population data. eLife 5, e10989 (2016).

31. Bosco, A., Breveglieri, R., Filippini, M., Galletti, C. \& Fattori, P. Reduced neural representation of arm/hand actions in the medial posterior parietal cortex. Sci. Rep. 9, 936 (2019).

32. Gold, J. I. \& Shadlen, M. N. The neural basis of decision making. Annu Rev Neurosci 30, 535-574 (2007).

33. Cisek, P., Puskas, G. A. \& El-Murr, S. Decisions in changing conditions: the urgencygating model. J Neurosci 29, 11560-11571 (2009).

34. Thura, D. \& Cisek, P. Deliberation and commitment in the premotor and primary motor cortex during dynamic decision making. Neuron 81, 1401-1416 (2014).

35. Ferrari-Toniolo, S., Visco-Comandini, F., Papazachariadis, O., Caminiti, R. \& BattagliaMayer, A. Posterior Parietal Cortex Encoding of Dynamic Hand Force Underlying Hand-Object Interaction. J. Neurosci. 35, 10899-10910 (2015).

36. Georgopoulos, A. P., Schwartz, A. B. \& Kettner, R. E. Neuronal population coding of movement direction. Science 233, 1416-1419 (1986).

37. Georgopoulos, A. P., Kalaska, J. F., Caminiti, R. \& Massey, J. T. On the relations between the direction of two-dimensional arm movements and cell discharge in primate motor cortex. J. Neurosci. Off. J. Soc. Neurosci. 2, 1527-1537 (1982).

38. Ashe, J. \& Georgopoulos, A. P. Movement parameters and neural activity in motor cortex and area 5. Cereb. Cortex 4, 590-600 (1994).

39. Evarts, E. V. Relation of pyramidal tract activity to force exerted during voluntary movement. J. Neurophysiol. 31, 14-27 (1968).

40. Georgopoulos, A. P., Caminiti, R., Kalaska, J. F. \& Massey, J. T. Spatial coding of movement: a hypothesis concerning the coding of movement direction by motor cortical populations. Exp. Brain Res. 49, 327-336 (1983).

41. Dann, B., Michaels, J. A., Schaffelhofer, S. \& Scherberger, H. Uniting functional network topology and oscillations in the fronto-parietal single unit network of behaving primates. eLife 5, e15719 (2016).

42. Pezzulo, G. \& Ognibene, D. Proactive Action Preparation: Seeing Action Preparation as a Continuous and Proactive Process. Motor Control 16, 386-424 (2011).

43. Pezzulo, G. Studying mirror mechanisms within generative and predictive architectures for joint action. Cortex 49, 2968-2969 (2013). 
bioRxiv preprint doi: https://doi.org/10.1101/2020.12.23.424150; this version posted December $23,2020$. The copyright holder for this preprint (which was not certified by peer review) is the author/funder, who has granted bioRxiv a license to display the preprint in perpetuity. It is made available under aCC-BY-NC-ND 4.0 International license.

44. Shen, L. \& Alexander, G. E. Preferential representation of instructed target location versus limb trajectory in dorsal premotor area. J. Neurophysiol. 77, 1195-1212 (1997).

45. Cisek, P. Neural representations of motor plans, desired trajectories, and controlled objects. Cogn. Process. 6, 15-24 (2005).

46. Kraskov, A., Dancause, N., Quallo, M. M., Shepherd, S. \& Lemon, R. N. Corticospinal neurons in macaque ventral premotor cortex with mirror properties: a potential mechanism for action suppression? Neuron 64, 922-930 (2009).

47. Georgopoulos, A. P., Kettner, R. E. \& Schwartz, A. B. Primate motor cortex and free arm movements to visual targets in three-dimensional space. II. Coding of the direction of movement by a neuronal population. J. Neurosci. Off. J. Soc. Neurosci. 8, 2928-2937 (1988). 48. Batschelet, E. Circular statistics in biology. Acad. PRESS 111 FIFTH AVE N. Y. NY 10003 1981388 (1981).

49. Thura, D. \& Cisek, P. Modulation of premotor and primary motor cortical activity during volitional adjustments of speed-accuracy trade-offs. J. Neurosci. 36, 938-956 (2016). 


\title{
SUPPLEMENTARY MATERIALS
}

Shared population-level dynamics in monkey premotor cortex during solo action, joint action and action observation

\author{
Giovanni Pezzulo ${ }^{1 *}$, Francesco Donnarumma $^{1 *}$, Simone Ferrari-Toniolo ${ }^{3,}{ }^{\ddagger}$, Paul Cisek $^{2}$, \\ Alexandra Battaglia-Mayer ${ }^{3}$
}

1. Institute of Cognitive Sciences and Technologies, National Research Council, 00185 Rome, Italy

2. Department of Physiology and Pharmacology, SAPIENZA University of Rome, 00185 Rome, Italy

3. Department of Neuroscience, University of Montreal, Canada

* These authors contributed equally to this paper.

‡ Current address: Department of Physiology, Development and Neuroscience, University of Cambridge, Cambridge CB2 3EG, UK

Correspondence to:

Alexandra Battaglia-Mayer (alexandra.battagliamayer@uniroma1.it)

Giovanni Pezzulo (giovanni.pezzulo@istc.cnr.it) 


\section{Example data from single monkeys}

In the main analyses, we constructed a neural population using data pooled from 2 monkeys and 36 sessions. The pooling procedure from two animals was motivated by the fact that they were tested together, either when acting jointly, or when one acted and the other observed, and also because when individual data sets were analysed, similar results were obtained between the two. We observed minor differences in the rank and coding of the principal components. Examples of single-monkey analyses are reported in Fig. S1. It is noting the similarity of neural dynamics. While the sign of PC X2 is inverted in monkey $\mathrm{K}$, the sign is arbitrary in PCA and only the relative magnitudes are meaningful ${ }^{1}$ when studying the overall patterns of neural dynamics.

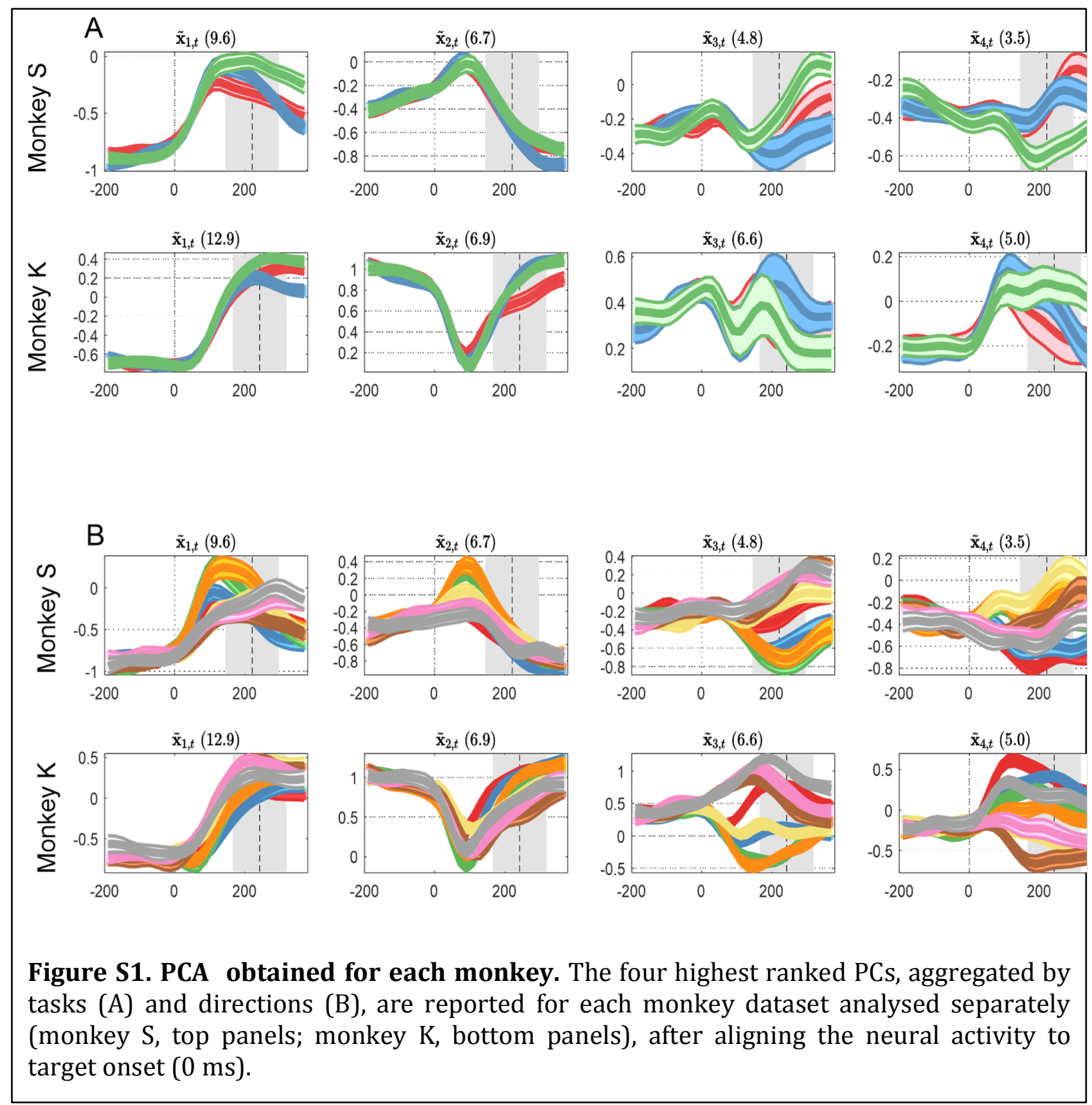




\section{Control analysis: eye movement task}

We performed a control analysis to rule out the possibility that the common pattern of directional gradient observed in the three tasks was mainly related to eye behavior, which was virtually identical in the SOLO, TOGETHER and OBS-OTHER tasks 2,3. For this, we conducted a separate PCA analysis that considered data from the three aforementioned tasks along with a fourth, control saccadic task (EYE). In the EYE task, monkeys had to perform saccadic movements from the central target to one of the 8 visual stimuli presented in same locations as those used in the SOLO, TOGETHER and OBS-OTHER. Neural activity in the EYE task was recorded from the same population as the other three tasks; but while the latter were performed interminging the different types of trials in one block, the saccadic trials were presented in a separate block, before or after the performace of the block of SOLO, TOGETHER and OBS-OTHER trials. For this new PCA, the neural activity was aligned to target onset.

Fig. S2A shows the four highest-ranked principal components X1-X4 obtained by clustering across task conditions (SOLO, OBS-OTHER, TOGETHER, and EYE). It shows that the neural representation of the EYE task is markedly different from the other three tasks, despite their similar oculomotor demands. The first principal component of PCA, which explains almost one third of the variance (X1, $30.7 \%$ ), clearly distinguishes EYE from the other three tasks, even before target onset; whereas the other three tasks are closely grouped together. This component may therefore discriminate only the tasks conditions performed in separate blocks. However, the other high-ranked components X2-X4 also clearly discriminate the saccadic (EYE) trials from the others, which show similar temporal trends.

Fig. S2B shows the four highest-ranked principal components X1-X4 obtained by clustering across directions (D1-D8). In the first principal component of PCA, the spatial features of the tasks are smeared out. Finally, although single unit activity recorded during the saccadic task was directionally tuned ${ }^{2,3}$, in PCA space the activity during the EYE task did not produce the systematic directional gradient (Figure S3D) that was clearly observed in the other tasks (Figure S3A-C).

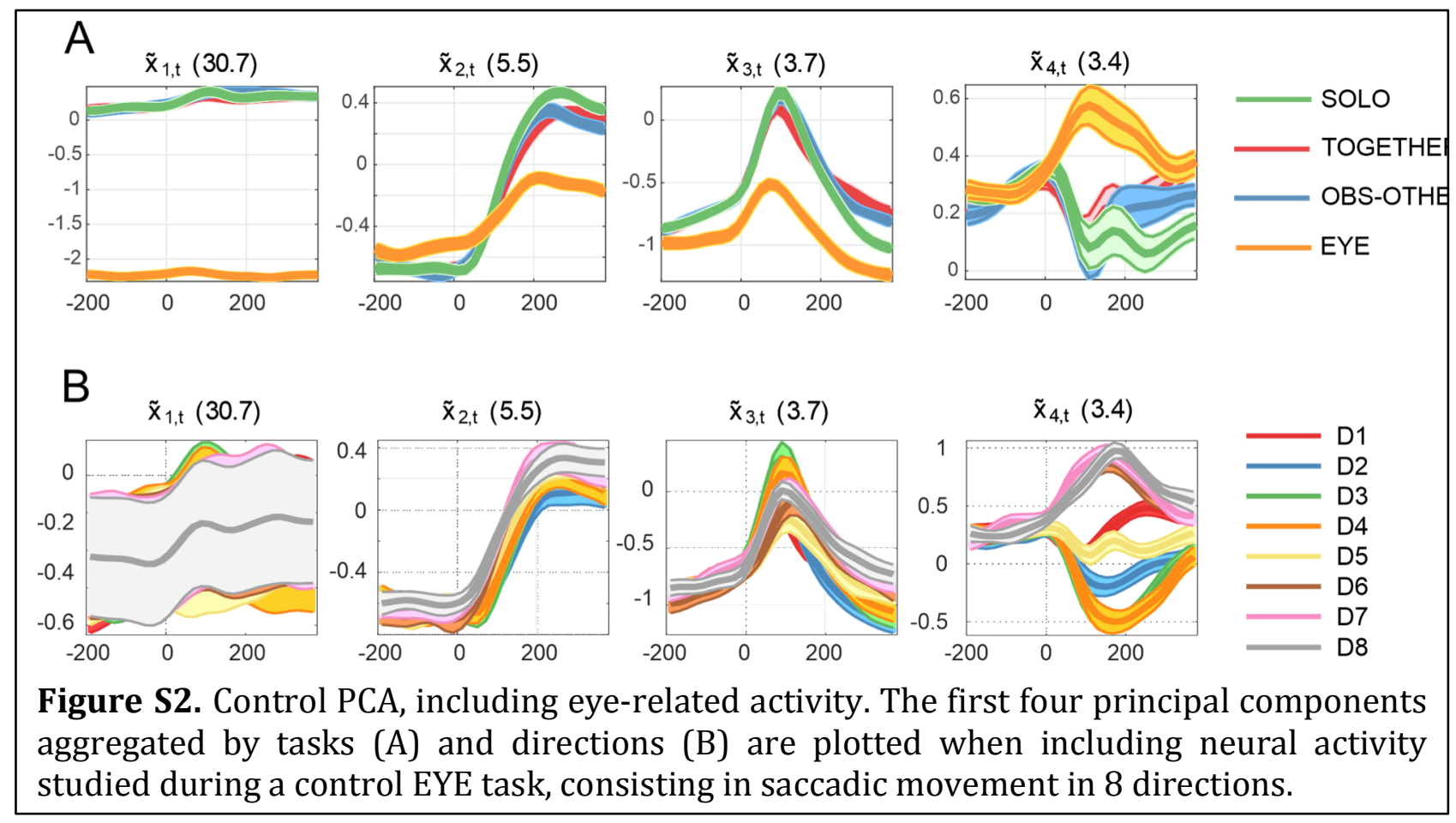




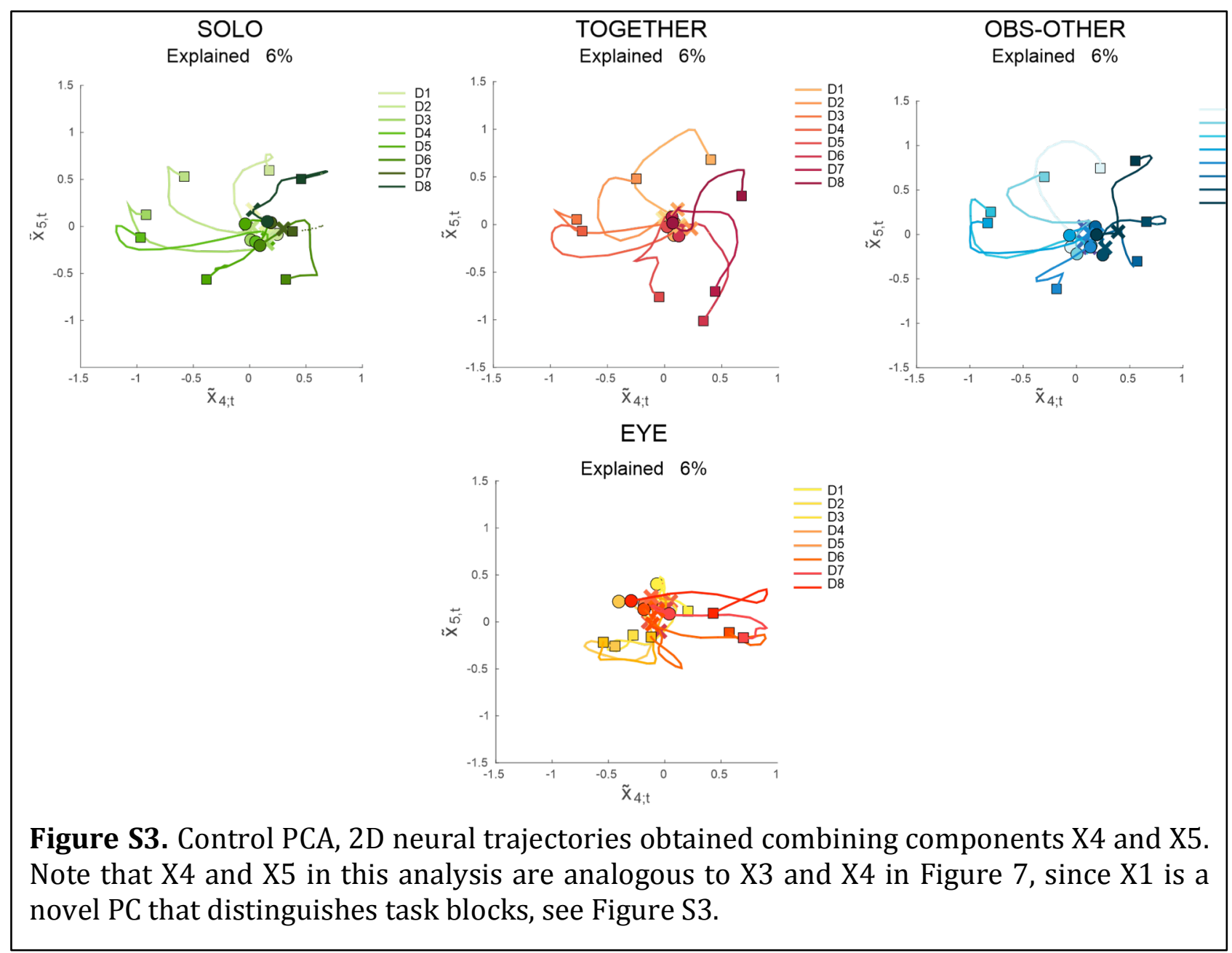

\section{References}

1. Jolliffe, I. T. \& Cadima, J. Principal component analysis: a review and recent developments. Philos. Transact. A Math. Phys. Eng. Sci. 374, (2016).

2. Ferrari-Toniolo, S., Visco-Comandini, F. \& Battaglia-Mayer, A. Two brains in action: Joint-action coding in the primate frontal cortex. J. Neurosci. 1512-18 (2019) doi:10.1523/JNEUROSCI.1512-18.2019.

3. Visco-Comandini, F. et al. Do non-human primates cooperate? Evidences of motor coordination during a joint action task in macaque monkeys. Cortex 70, 115-127 (2015). 